\title{
THE ASYMPTOTIC BEHAVIOR OF HOLOMORPHIC 1-COCHAINS
}

\author{
DAISUKe YAMAKI
}

\begin{abstract}
In this paper, we focus on relations between holomorphic 1-forms and holomorphic 1-cochains on a closed Riemann surface. Holomorphic 1-cochains are defined by Wilson in 2008 using a combinatorial Hodge theory. A holomorphic 1-form is characterized by its periods. So is a holomorphic 1-cochain. We consider relations between a holomorphic 1-form and a holomorphic 1-cochain which have the same periods and show that holomorphic 1-cochains provide an approximation of holomorphic 1-forms.
\end{abstract}

\section{Introduction}

In this paper, we show that holomorphic 1-cochains provide an approximation to holomorphic 1-forms on closed Riemann surfaces with triangulations.

Holomorphic 1-cochains are defined by Wilson [10] as follows. Let $M$ be a closed Riemann surface of genus $g$ and $K$ a triangulation of $M$. Then the space $\mathscr{H} C^{1,0}(K)$ of holomorphic 1 -cochains is defined as the span of the eigenvectors for non-positive imaginary eigenvalues of the combinatorial star operator $\star$ introduced in [9]. Further, for a canonical homology basis $\Sigma=\left\{a_{1}, \ldots, a_{g}, b_{1}, \ldots, b_{g}\right\}$ of $M$, Wilson defined the combinatorial periods of a holomorphic 1-cochain $\sigma$ by the complex numbers $\sigma\left(a_{j}\right)$ and $\sigma\left(b_{j}\right)$, and showed that holomorphic 1-cochains satisfy Riemann's bi-linear relation. Also, the relation gives a unique basis $\left\{\sigma_{1}, \ldots, \sigma_{g}\right\}$ of $\mathscr{H} C^{1,0}(K)$, which satisfies $\sigma_{j}\left(a_{k}\right)=\delta_{j k}$ and is called the canonical basis. Using the canonical basis, Wilson defined the combinatorial period matrix of holomorphic 1-cochains and showed that the combinatorial period matrix converges to the (conformal) period matrix, as the mesh of the triangulation tends to zero. However, this convergence result dose not imply that holomorphic 1-cochains converge to holomorphic 1-forms. As the main result of this paper, we show that holomorphic 1-cochains also converge to holomorphic 1-forms in Theorem 5.3.

Our work is based on the results of Dodziuk and Patodi $[1,2]$. They showed that by using the Whitney map from cochains into differential forms, cochains provide a good approximation to smooth differential forms. Their results yields

2010 Mathematics Subject Classification. Primary 57R12; Secondary 30F30.

Key words and phrases. Holomorphic 1-cochain, holomorphic 1-form.

Received July 28, 2015; revised September 29, 2015. 
many convergence results. For instance, Dodziuk and Patodi proved that Eckmann's Hodge decompositions of cochains, given by the Whitney inner product, converge to the Hodge decompositions of smooth differential forms in $[1,2]$, Wilson showed that the combinatorial star operator $\star$ converges to the smooth Hodge star operator in [9]. A convergence result of combinatorial period matrices was also given in [10].

To induce Theorem 5.3, we show three theorems which are Theorem 5.4, 5.6 and 5.7. Then, by combining these theorems, we obtain Theorem 5.3.

In Theorem 5.4, for a fixed triangulation, we estimate the differences between holomorphic 1-cochains and holomorphic 1-forms using a matrix equation, which implies the difference of the period matrix and the combinatorial period matrix of a fixed triangulation. Then we obtain a vector $\Phi_{K}=\left(\varphi_{1}, \ldots, \varphi_{g}\right) \in(0,1]^{g}$ such that $\left\langle\star \sigma_{j}, \sigma_{j}\right\rangle_{C}=\left\langle-i \varphi_{j} \sigma_{j}, \sigma_{j}\right\rangle_{C}$, where $\langle,\rangle_{C}$ is the Whitney inner product on the cochains. Note that since the space $\mathscr{H} C^{1,0}(K)$ is the span of the eigenvectors for non-positive imaginary eigenvalues of $\star$, the canonical basis $\left\{\sigma_{1}, \ldots, \sigma_{g}\right\}$ is not eigenbasis of $\star$ in general. Using the vector $\Phi_{K}$ and the combinatorial period matrix, we have the estimation of differences between holomorphic 1-cochains and holomorphic 1-forms for a fixed triangulation.

Finally we study the behavior of $\Phi_{K}$. More precisely, we show that $\Phi_{K}$ is always equal to 1 for any triangulation of a complex torus in Theorem 5.6, and in the case of $g>1, \Phi_{K}$ converges to $(1, \ldots, 1)$, as the mesh tend to zero in Theorem 5.7.

\section{Combinatorial Hodge theory}

In this section, we recall the construction of a combinatorial Hodge theory. Let $M$ be a closed smooth Riemannian $n$-manifold, and let $\Omega^{j}(M)$ be the space of smooth $j$-forms on $M$ with the exterior derivative $d$ and the Hodge star operator $\star$. Then we introduce an inner product $\langle,\rangle_{\Omega}$ on $\Omega(M)$ which is given by the Riemannian metric of $M$. Then we define $d^{*}:=(-1)^{j(j+1-n)} \star d \star$ and the space $\mathscr{H} \Omega^{j}(M)$ of harmonic $j$-forms on $M$ by

$$
\mathscr{H} \Omega^{j}(M)=\left\{\omega \in \Omega^{j}(M) \mid d \omega=d^{*} \omega=0\right\},
$$

and have the Hodge decomposition

$$
\Omega^{j}(M)=d \Omega^{j-1}(M) \oplus \mathscr{H} \Omega^{j}(M) \oplus d^{*} \Omega^{j+1}(M) .
$$

Let $K$ be a $C^{\infty}$ triangulation of $M$. Now we identify $|K|$ and $M$ and fix an ordering of the vertices of $K$. Then we denote the $i$-th vertex of $K$ by $p_{i}$ and the barycentric coordinate corresponding to $p_{i}$ by $\mu_{i}$. Let $C^{j}(K)$ be the simplicial $j$-cochains of $K$ with values in $\mathbf{R}$. Given the ordering of vertices, we have a coboudary operator $\delta: C^{j}(K) \rightarrow C^{j+1}(K)$. Since $K$ is a finite complex, we can identify chains and cochains and then for $\sigma \in C^{j}(K)$, we may write

$$
\sigma=\sum_{\tau} c_{\tau} \cdot \tau
$$


where $c_{\tau} \in \mathbf{R}$ and the sum is taken over all $j$-simplices of $K$. We write $\tau=$ $\left[p_{0}, p_{1}, \ldots, p_{j}\right]$ of $K$ with the vertices in an increasing sequence with respect to the ordering of vertices in $K$.

Definition 2.1. For a triangulation $K$, We define the mesh $\eta(K)$ of $K$ by

$$
\eta(K)=\sup r(p, q)
$$

where $r$ means the geodesic distance in $M$ and the supremum is taken over all pairs of vertices $p, q$ of a 1 -simplex in $K$.

We define the fullness $\Theta(K)$ of $K$ by

$$
\Theta(K)=\inf \frac{\operatorname{vol}(\sigma)}{\eta(K)^{n}},
$$

where the inf is taken over all $n$-simplices $\sigma$ of $K$ and $\operatorname{vol}(\sigma)$ is the Riemannian volume of $\sigma$, as a Riemannian submanifold of $M$.

Then we may consider only triangulations whose fullness are bounded below by some positive real constant, see $[2,8,9,10]$ for details. Here we assume that all triangulations satisfy this condition. This means that the shapes of all simplices in $K$ do not too thin.

Now we assume that the cochains $C(K)$ are equipped with a non-degenerate inner product $\langle,\rangle_{C}$ such that $C^{j}(K) \perp C^{k}(K)$ for $j \neq k$. Then we define the adjoint operator of $\delta$ :

Definition 2.2. The adjoint operator $\delta^{*}: C^{j}(K) \rightarrow C^{j-1}(K)$ of $\delta$ is defined by $\left\langle\delta^{*} \sigma_{1}, \sigma_{2}\right\rangle_{C}=\left\langle\sigma_{1}, \delta \sigma_{2}\right\rangle_{C}$.

Then two operators $\delta$ and $\delta^{*}$ give rise to the harmonic cochains as follows.

Definition 2.3. We define the space $\mathscr{H} C^{j}(K)$ of harmonic $j$-cochains of $K$ by

$$
\mathscr{H} C^{j}(K)=\left\{\sigma \in C^{j}(K) \mid \delta \sigma=\delta^{*} \sigma=0\right\} .
$$

Eckmann showed that an inner product $\langle,\rangle_{C}$ provides the Hodge decomposition of cochains.

THEOREM $2.4([4])$. There is an orthogonal direct sum decomposition

$$
C^{j}(K)=\delta C^{j-1}(K) \oplus \mathscr{H} C^{j}(K) \oplus \delta^{*} C^{j+1}(K)
$$

and $\mathscr{H} C^{j}(K) \cong H^{j}(K)$, the cohomology of $(K, \delta)$ in degree $j$.

In $[1,2,9]$, the relations between the Hodge theory of smooth forms and the combinatorial Hodge thery of cochains were studied. Now we recall some 
results related to these relations. To describe, we define two maps between differential forms and cochains. First, we define a map $W$ from $C^{j}(K)$ into $\mathscr{L}^{2} \Omega^{j}(M)$ which is the completion of $\Omega^{j}(M)$ with respect to $\langle,\rangle_{\Omega}$. The map $W$ is called the Whitney map.

Definition 2.5. For a $j$-simplex $\tau=\left[p_{0}, \ldots, p_{j}\right]$ of $K$, we define $W \tau$ by

$$
W \tau=j ! \sum_{i=0}^{j}(-1)^{i} \mu_{i} d \mu_{0} \wedge \cdots \wedge \widehat{d \mu}_{i} \wedge \cdots \wedge d \mu_{j},
$$

where ${ }^{\wedge}$ over a symbol means deletion. $\quad W$ is defined on $C(K)=\bigoplus_{j \in\{0,1,2\}} C^{j}(K)$ by extending linearly.

Remark 2.6. The barycentric coordinates $\mu_{j}$ are not even of class $C^{1}$, but they are of class $C^{\infty}$ on the interior of any simplex of $K$. This implies that $d \mu_{j}$ is defined and $W \tau$ is well-defined. Therefore $d W$ is also well-defined on $\mathscr{L}^{2} \Omega^{j}(M)$.

The Whitney map $W$ has several properties.

Proposition 2.7 ([8]). The following hold:

(1) $W \tau=0$ on $M \backslash \overline{S t(\tau)}$,

(2) $d W=W \delta$,

where $\overline{\operatorname{St}(\tau)}$ is the closure of the open star $\operatorname{St}(\tau)$.

Next we define the de Rham map $R$ from differential forms to cochains which is given by integration:

Definition 2.8. For any differential form $\omega$ and chain $c$, the de Rham map $R$ is defined by

$$
R \omega(c)=\int_{c} \omega
$$

The de Rham map is a chain map:

LEMMA 2.9 ([3]). The following holds:

$$
\delta R=R d .
$$

The Whitney map and the de Rham map satisfy the following relation, see $[1,2,8]$ :

THEOREM 2.10. The following holds:

$$
R W=I d .
$$


In general, $W R \neq I d$. However, Dodziuk and Patodi [2] showed the following approximation theorem.

THEOREM 2.11 ([1]). There exist a positive constant $C$ and a positive integer $m$, independent of $K$, such that

$$
\|\omega-W R \omega\|_{\Omega} \leq C \cdot\left\|(I d+\Delta)^{m} \omega\right\|_{\Omega} \cdot \eta(K)
$$

for all $C^{\infty}$ differential forms $\omega$ on $M$.

This implies that for a fine triangulation, cochains provide a good approximation to differential forms. Also, Dodziuk and Patodi studied the Hodge decomposition of cochains, given by the Whitney inner product. The Whitney inner product $\langle,\rangle_{C}$ is defined by

$$
\langle\sigma, \tau\rangle_{C}=\langle W \sigma, W \tau\rangle_{\Omega}
$$

for $\sigma, \tau \in C(K)$ and it is proven in [1] that the Whitney inner product is nondegenerate. Then Dodziuk and Patodi $[1,2]$ showed that the Hodge decomposition of cochains, given by the Whitney inner product, is an approximation of the Hodge decomposition of (smooth) forms.

Theorem 2.12. Let $\omega \in \Omega^{j}(M)$ and $R \omega \in C^{j}(K)$ have Hodge decompositions

$$
\begin{aligned}
\omega & =d \omega_{1}+\omega_{2}+d^{*} \omega_{3} \\
R \omega & =\delta a_{1}+a_{2}+\delta^{*} a_{3} .
\end{aligned}
$$

Then

$$
\begin{array}{r}
\left\|d \omega_{1}-W \delta a_{1}\right\|_{\Omega} \leq \lambda \cdot\left\|(I d+\Delta)^{m} \omega\right\|_{\Omega} \cdot \eta(K) \\
\left\|\omega_{2}-W a_{2}\right\|_{\Omega} \leq \lambda \cdot\left\|(I d+\Delta)^{m} \omega\right\|_{\Omega} \cdot \eta(K) \\
\left\|d^{*} \omega_{3}-W \delta^{*} a_{3}\right\|_{\Omega} \leq \lambda \cdot\left\|(I d+\Delta)^{m} \omega\right\|_{\Omega} \cdot \eta(K)
\end{array}
$$

where $\lambda$ and $m$ are independent of $\omega$ and $K$.

In [9], Wilson defined the combinatorial Hodge star oprator on cochains. To define it, he used the following cup product on cochains, which is defined by Whitney [8]:

Definition 2.13. We define $U: C^{j}(K) \otimes C^{k}(K) \rightarrow C^{j+k}(K)$ by

$$
\sigma \cup \tau=R(W \sigma \wedge W \tau)
$$

for $\sigma \in C^{j}(K)$ and $\tau \in C^{k}(K)$. 
Then Wilson defined the star operator as follows:

Definition 2.14. Let $\langle,\rangle_{C}$ be a positive definite inner product on $C(K)$ such that $C^{j}(K) \perp C^{k}(K)$ for $j \neq k$. For $\sigma \in C^{j}(K)$, we define $\star \sigma \in C^{n-j}(K)$ by

$$
\langle\star \sigma, \tau\rangle_{C}=(\sigma \cup \tau)[M],
$$

where $[M]$ denotes the fundamental class of $M$.

This star operator $\star$ has several properties:

LEMma 2.15 ([9]). The following hold:

(1) $\star \delta=(-1)^{j+1} \delta^{*} \star$, i.e. $\star$ is a chain map.

(2) For $\sigma \in C^{j}(K)$ and $\tau \in C^{n-j}(K),\langle\star \sigma, \tau\rangle_{C}=(-1)^{j(n-j)}\langle\sigma, \star \tau\rangle_{C}$, i.e. $\star$ is (graded) skew-adjoint.

(3) $\star$ induces isomorphisms $\mathscr{H} C^{j}(K) \rightarrow \mathscr{H} C^{n-j}(K)$ on harmonic cochains.

Using Theorem 2.11, Wilson showed that $\star$ converges to the Hodge star operator $\star$ on $\Omega(M)$ :

THEOREM 2.16 ([9]). There exist a positive constant $C$ and a positive integer $m$, independent of $K$, such that

$$
\|\star \omega-W \star R \omega\|_{\Omega} \leq C \cdot\left\|(I d+\Delta)^{m} \omega\right\|_{\Omega} \cdot \eta(K),
$$

for all $C^{\infty}$ differential forms $\omega$ on $M$.

Under the assumption that the cochains $C(K)$ are equipped with the Whitney inner product, Wilson also showed that $\star$ respects the Hodge decomposition of $C(K)$ and $\Omega(M)$ :

TheOREM 2.17 ([9]). Let $\omega \in \Omega^{j}(M)$ and $R \omega \in C^{j}(K)$ have the Hodge decompositions

$$
\begin{aligned}
\omega & =d \omega_{1}+\omega_{2}+d^{*} \omega_{3} \\
R \omega & =\delta a_{1}+a_{2}+\delta^{*} a_{3} .
\end{aligned}
$$

Then there exist a positive constant $C$ and a positive integer $m$, independent of $K$, such that

$$
\begin{aligned}
\left\|\star d \omega_{1}-W \star \delta a_{1}\right\|_{\Omega} & \leq C \cdot\left(\left\|(I d+\Delta)^{m} \omega\right\|_{\Omega}+\left\|(I d+\Delta)^{m} d \omega_{1}\right\|_{\Omega}\right) \cdot \eta(K), \\
\left\|\star \omega_{2}-W \star a_{2}\right\|_{\Omega} & \leq C \cdot\left(\left\|(I d+\Delta)^{m} \omega\right\|_{\Omega}+\left\|(I d+\Delta)^{m} \omega_{2}\right\|_{\Omega}\right) \cdot \eta(K), \\
\left\|\star d^{*} \omega_{3}-W \star \delta^{*} a_{3}\right\|_{\Omega} & \leq C \cdot\left(\left\|(I d+\Delta)^{m} \omega\right\|_{\Omega}+\left\|(I d+\Delta)^{m} d^{*} \omega_{3}\right\|_{\Omega}\right) \cdot \eta(K) .
\end{aligned}
$$




\section{Holomorphic 1-forms on Riemann surfaces}

In this section, we recall the definitions and properties of holomorphic 1 -forms and period matrices. Let $M$ be a closed Riemann surface of genus $g>0$, $\Omega^{j}(M)$ be the space of smooth $j$-forms on $M$ with values in $\mathrm{C}$ and $\Omega(M)=$ $\bigoplus_{j \in\{0,1,2\}} \Omega^{j}(M)$. Using the Hodge star operator $\star$, the space of holomorphic 1 -forms on $M$ is defined by

$$
\mathscr{H} \Omega^{1,0}(M)=\left\{\omega \in \mathscr{H} \Omega^{1}(M) \mid \star \omega=-i \omega\right\} .
$$

Now we define an inner product $\langle,\rangle_{\Omega}$ on $\Omega(M)$ by

$$
\left\langle\omega_{1}, \omega_{2}\right\rangle_{\Omega}=\iint_{M} \omega_{1} \wedge \star \overline{\omega_{2}} .
$$

Next we take a homology basis $\Sigma=\left\{a_{1}, \ldots, a_{g}, b_{1}, \ldots, b_{g}\right\}$ for the first homology, which satisfies the following properties: the intersection of any two basis elements is non-zero only for $a_{j}$ and $b_{j}$, in which case it is equal to one. We say that such a basis is canonical. Then we define periods of a closed 1-form $\omega$ with respect to $\Sigma$ by

$$
\int_{a_{j}} \omega, \quad \int_{b_{k}} \omega
$$

for $1 \leq j, k \leq g$. These periods satisfy the following relations, which are called Riemann's bi-linear relations: for two closed 1-forms $\omega_{1}, \omega_{2}$,

$$
\left\langle\star \omega_{1}, \omega_{2}\right\rangle_{\Omega}=\sum_{j=1}^{g}\left(\int_{a_{j}} \omega_{1} \int_{b_{j}} \overline{\omega_{2}}-\int_{b_{j}} \omega_{1} \int_{a_{j}} \overline{\omega_{2}}\right) .
$$

Especially, for any $\omega_{1}, \omega_{2} \in \mathscr{H} \Omega^{1,0}(M)$,

$$
\sum_{j=1}^{g}\left(\int_{a_{j}} \omega_{1} \int_{b_{j}} \omega_{2}-\int_{b_{j}} \omega_{1} \int_{a_{j}} \omega_{2}\right)=0
$$

This relation inplies that if all $\int_{a_{j}} \omega$ of a holomorphic 1-form $\omega$ are zero, then $\omega=0$. By the rank-nullity theorem for linear maps, we have the following isomorphism:

$$
\mathscr{H} \Omega^{1,0}(M) \ni \omega \mapsto\left(\int_{a_{1}} \omega, \ldots, \int_{a_{g}} \omega\right) \in \mathbf{C}^{g} .
$$

Namely, holomorphic 1-forms are characterized by $A$-periods. Using this property, we define a unique basis $\left\{\theta_{1}, \ldots, \theta_{g}\right\}$ of $\mathscr{H} \Omega^{1,0}(M)$ which satisfies $\int_{a_{k}} \theta_{j}=\delta_{j k}$ for $1 \leq j, k \leq g$. We call this basis the canonical basis of $\mathscr{H} \Omega^{1,0}(M)$ (determined by a canonical homology basis $\Sigma$ ). Then we define the period matrix $\Pi=\left(\pi_{j k}\right)_{1 \leq j, k \leq g}$ by $\pi_{j k}=\int_{b_{k}} \theta_{j}$. It is known that period matrices lie in the Siegel upper half space. 


\section{Holomorphic 1-cochains and combinatorial period matrices}

Wilson applied the combinatorial Hodge theory to Riemann surfaces in [10]. To define holomorphic 1-cochains, we need to extend some of the definitions of the combinatorial Hodge theory to the complex setting. Let $M$ be a closed Riemann surface with a triangulation $K$, and let $C^{j}(K)$ be the simplicial cochains with values in $\mathbf{C}$. Here suppose that the cochains $C(K)$ are equipped with a non-degenerate inner product such that $C^{i}(K)$ and $C^{j}(K)$ are orthogonal for $i \neq j$. Then the star operator $\star$ on cochains $C(K)$ is defined by

$$
\langle\star \sigma, \tau\rangle_{C}=(\sigma \cup \bar{\tau})[M],
$$

where $U$ is extended over $\mathbf{C}$ linearly. Also, for complex cochains, we have a Hodge decomposition

$$
C^{1}(K)=\delta C^{0}(K) \oplus \mathscr{H} C^{1}(K) \oplus \delta^{*} C^{2}(K) .
$$

By Lemma 2.15 , we regard $\star$ as an isomorphism from $\mathscr{H} C^{1}(K)$ into $\mathscr{H} C^{1}(K)$ which is skew-adjoint. Then we define holomorphic 1 -cochains as follows:

Definition 4.1. Let $\langle,\rangle_{C}$ be a hermitian inner product on the complex valued simpicial 1-cochains which is $\mathbf{R}$-valued on $\mathbf{R}$-cochains. We define the space $\mathscr{H} C^{1,0}(K)$ of holomorphic 1 -cochains to be the span of the eigenvectors for non-positive imaginary eigenvalues of $\star$ and the space $\mathscr{H} C^{0,1}(K)$ of antiholomorphic 1-cochains to be the span of the eigenvectors for non-negative imaginary eigenvalues of $\star$.

Now we assume that the cochains $C(K)$ are equipped with the Whitney inner product. Note that the Whitney inner product is $\mathbf{R}$-valued on $\mathbf{R}$-cochains. Then we have the following properties of $\mathscr{H} C^{1,0}(K)$ and $\mathscr{H} C^{0,1}(K)$, due to [7, 10].

Lemma 4.2. Let $M$ be a closed Riemann surface of genus $g$ with a canonical homology basis $\Sigma$ and a triangulation $K$. Then, the following hold:

(1) $\mathscr{H} C^{1}(K)=\mathscr{H} C^{1,0}(K) \oplus \mathscr{H} C^{0,1}(K)$.

(2) $\operatorname{dim}_{\mathbf{C}} \mathscr{H} C^{1,0}(K)=\operatorname{dim}_{\mathbf{C}} \mathscr{H} C^{0,1}(K)=g$.

(3) Complex conjugation maps $\mathscr{H} C^{1,0}(K)$ to $\mathscr{H} C^{0,1}(K)$ and vice versa.

Next we define combinatorial periods.

Definition 4.3. Let $M$ be a closed Riemann surface of genus $g$ with a canonical homology basis $\Sigma$ and a triangulation $K$. We define the combinatorial periods of $\sigma \in \mathscr{H} C^{1}(K)$ by the following complex numbers:

$$
\sigma\left(a_{j}\right), \sigma\left(b_{j}\right) \text { for } 1 \leq j \leq g .
$$


Especially, combinatorial periods of holomorphic 1-cochains also satisfy Riemann's bi-linear relations.

TheOREM 4.4 ([10]). For $\sigma, \tau \in \mathscr{H} C^{1,0}(K)$, we have

$$
\sum_{j=1}^{g}\left(\sigma\left(a_{j}\right) \tau\left(b_{j}\right)-\sigma\left(b_{j}\right) \tau\left(a_{j}\right)\right)=0 .
$$

To define combinatorial period matrices, Wilson [10] showed the following relation. For $\sigma, \tau \in \mathscr{H} C^{1,0}(K)$,

$$
\langle\star \sigma, \tau\rangle_{C}=\sum_{j=1}^{g}\left(\sigma\left(a_{j}\right) \overline{\tau\left(b_{j}\right)}-\sigma\left(b_{j}\right) \overline{\tau\left(a_{j}\right)}\right) .
$$

This yields the following.

COROLlaRy $4.5([10])$. Let $\sigma$ be a holomorphic 1-cochain.

(1) If all A-periods $\sigma\left(a_{j}\right), 1 \leq j \leq g$ or all B-periods $\sigma\left(b_{j}\right), 1 \leq j \leq g$ are vanish, then $\sigma=0$.

(2) If all A-periods $\sigma\left(a_{j}\right), 1 \leq j \leq g$ and all B-periods $\sigma\left(b_{j}\right), 1 \leq j \leq g$ are real, then $\sigma=0$.

For any basis $\left\{\tau_{1}, \ldots, \tau_{g}\right\}$ for $\mathscr{H} C^{1,0}(K)$, we consider the following equation of $\left(c_{i j}\right)_{1 \leq i, j \leq g}$ :

$$
\sum_{i=1}^{g} c_{i j} \tau_{i}\left(a_{k}\right)=\delta_{j k}
$$

By Corollary $4.5(1)$, the matrix $\left(c_{i j}\right)_{1 \leq i, j \leq g}$ is uniquely determined by a triple $(M, \Sigma, K)$. Then we put $\sigma_{j}=\sum_{j=1}^{g} c_{i j} \tau_{i}$. This basis $\left\{\sigma_{1}, \ldots, \sigma_{g}\right\}$ is called the canonical basis of $\mathscr{H} C^{1,0}(K)$. follows.

Using the canonical basis, we define combinatorial period matrices as

Definition 4.6. Let $M$ be a closed Riemann surface of genus $g$ with a canonical homology basis $\Sigma$ and a triangulation $K$. Let $\left\{\sigma_{1}, \ldots, \sigma_{g}\right\}$ be the canonical basis of $\mathscr{H} C^{1,0}(K)$. Then the combinatorial period matrix $\Pi_{K}=$ $\left(\pi_{j k}^{K}\right)_{1 \leq j, k \leq g}$ of $M$ is defined by $\pi_{j k}^{K}=\sigma_{j}\left(b_{k}\right)$.

TheOREM 4.7 ([10]). Combinatorial period matrices are symmetric and their imaginary parts are positive definite.

This theorem implies that combinatorial period matrices lie in the Siegel upper half space. Now we recall some relations between period matrices and combinatorial period matrices. 
THEOREM 4.8 ([10]). Let $M$ be a closed Riemann surface with a canonical homology basis $\Sigma$, and let $\Pi$ be the period matrix. Let $\left\{K_{n}\right\}_{n \in \mathbf{N}}$ be a sequence of triangulations of $M$ with mesh converging to zero. Then the combinatorial period matrices $\Pi_{K_{n}}$ satisfy

$$
\lim _{n \rightarrow \infty} \Pi_{K_{n}}=\Pi
$$

To prove this theorem, Wilson showed the following lemmas, which are also used for the proof of Theorem 5.7.

Lemma 4.9 ([10]). Let $M$ be a closed Riemann surface with a triangulation $K$. For any $\omega \in \mathscr{H} \Omega^{1,0}(M)$ which has the following decomposition

$$
R \omega=\delta g+h_{1}+h_{2}+\delta^{*} k,
$$

where $h_{1} \in \mathscr{H} C^{1,0}(K)$ and $h_{2} \in \mathscr{H} C^{0,1}(K)$, there exists positive constant $C$, dependent on $\omega$ but independent of $K$, such that

$$
\left\|W h_{1}-\omega\right\|_{\Omega} \leq C \cdot \eta(K) .
$$
lemma:

Also, the original proof of the above lemma in [10] provides the following

Lemma 4.10. Let $M$ be a closed Riemann surface with a triangulation $K$. For any $\omega \in \mathscr{H} \Omega^{1,0}(M)$ which has the following decomposition

$$
R \omega=\delta g+h_{1}+h_{2}+\delta^{*} k
$$

where $h_{1} \in \mathscr{H} C^{1,0}(K)$ and $h_{2} \in \mathscr{H} C^{0,1}(K)$, there exists positive constant $C$, dependent on $\omega$ but independent of $K$, such that

$$
\left\|W \star h_{1}-\star \omega\right\|_{\Omega} \leq C \cdot \eta(K) .
$$

Proof. By Theorem 2.12 and 2.17, there is a constant $C$, indepedent of $K$, such that

$$
\begin{aligned}
C \cdot \eta(K) & \geq\left\|W \star\left(h_{1}+h_{2}\right)-\star \omega\right\|_{\Omega}+\left\|\omega-W\left(h_{1}+h_{2}\right)\right\|_{\Omega} \\
& =\left\|W \star\left(h_{1}+h_{2}\right)-\star \omega\right\|_{\Omega}+\left\|\star \omega+i W\left(h_{1}+h_{2}\right)\right\|_{\Omega} \\
& \geq\left\|W \star h_{1}+W \star h_{2}+i W\left(h_{1}+h_{2}\right)\right\|_{\Omega} \\
& =\left\|\star h_{1}+\star h_{2}+i\left(h_{1}+h_{2}\right)\right\|_{C} .
\end{aligned}
$$

Let $\phi_{1}, \ldots, \phi_{q}$ be an orthogonal eigenbasis of $\mathscr{H} C^{1,0}(K)$ for $\star$, with eigenvalues $-i \lambda_{1}, \ldots,-i \lambda_{g}\left(\lambda_{j}>0\right)$, and let $\tilde{\phi}_{1}, \ldots, \tilde{\phi}_{g}$ be an orthogonal eigenbasis of $\mathscr{H} C^{0,1}(K)$ for $\star$, with eigenvalues $i \tilde{\lambda}_{1}, \ldots, i \tilde{\lambda}_{g}\left(\tilde{\lambda}_{j}>0\right)$. Then we may write $h_{1}=\sum_{j=1}^{g} c_{j} \phi_{j}$ and $h_{2}=\sum_{j=1}^{g} \tilde{c}_{j} \tilde{\phi}_{j}$. Since $\mathscr{H} C^{1,0}(K)$ and $\mathscr{H} C^{0,1}(K)$ are orthogonal, we have 


$$
\begin{aligned}
C^{2} \cdot \eta(K)^{2} & \geq\left\|\sum_{j=1}^{g}\left(1-\lambda_{j}\right) c_{j} \phi_{j}\right\|_{C}^{2}+\left\|\sum_{j=1}^{g}\left(1+\tilde{\lambda}_{j}\right) \tilde{c}_{j} \tilde{\phi}_{j}\right\|_{C}^{2} \\
& =\sum_{j=1}^{g}\left(1-\lambda_{j}\right)^{2}\left|c_{j}\right|^{2}\left\|\phi_{j}\right\|_{C}^{2}+\sum_{j=1}^{g}\left(1+\tilde{\lambda}_{j}\right)^{2}\left|\tilde{c}_{j}\right|^{2}\left\|\tilde{\phi}_{j}\right\|_{C}^{2} \\
& \geq \sum_{j=1}^{g} \tilde{\lambda}_{j}^{2}\left|\tilde{c}_{j}\right|^{2}\left\|\tilde{\phi}_{j}\right\|_{C}^{2} \\
& =\left\|\star h_{2}\right\|_{C}^{2} .
\end{aligned}
$$

Hence we conclude

$$
\left\|W \star h_{1}-\star \omega\right\|_{\Omega} \leq\left\|W \star\left(h_{1}+h_{2}\right)-\star \omega\right\|_{\Omega}+\left\|\star h_{2}\right\|_{C} \leq 2 C \cdot \eta(K) .
$$

In the proof of Theorem 4.8 (Theorem 7.2 in [10]), for a sequence $\left\{K_{n}\right\}$ of triangulations with the mesh converging to zero and the holomorphic part $h_{j}^{n}$ of $R^{n} \theta_{j} \in C^{1}\left(K_{n}\right)$, Wilson stated that Lemma 4.9 (Lemma 7.1 in [10]) provides

$$
\lim _{n \rightarrow \infty} h_{j}^{n}\left(a_{k}\right)=\int_{a_{k}} \theta_{j}=\delta_{j k} .
$$

However, in [11], Wilson remarked that (4.1) dose not follows from the lemma since the convergence in the lemma is with respect to $\mathscr{L}^{2}$-norm, and the integration is not a bounded operator on smooth forms with respect to the norm. Also, Wilson stated that using the following lemma, (4.1) holds since we are considering smooth differential forms that are closed.

LEMma 4.11 ([11]). Let $\omega_{n}$ be a sequence of smooth closed differential forms on a closed Riemannian manifold which converge in $L^{2}$ to a smooth form $\omega$. Then for any cycle, the sequence $\int_{C} \omega_{n}$ converges to $\int_{C} \omega$.

In [12], for a fixed triangulation, we can find the difference of the period matrix and the combinatorial period matrix of a closed Riemann surface with a canonical homology basis.

THEOREM 4.12 ([12]). Let $M$ be a closed Riemann surface of genus $g$ with a canonical homology basis $\Sigma$ and a triangulation $K$, and let $\Pi$ be the period matrix and $\Pi_{K}$ the combinatorial period matrix. Let $\left\{\theta_{1}, \ldots, \theta_{g}\right\}$ be the canonical basis of $\mathscr{H} \Omega^{1,0}(M)$ and $\left\{\sigma_{1}, \ldots, \sigma_{g}\right\}$ the canonical basis of $\mathscr{H} C^{1,0}(K)$. Then the following equation holds:

$$
\Pi=\overline{\Pi_{K}}-\overline{\Lambda_{K}}
$$

where $\Lambda_{K}=\left(\left\langle W \sigma_{j}, \star \theta_{k}\right\rangle_{\Omega}\right)_{1 \leq j, k \leq g}$. 
Proposition 4.13 ([12]). For a closed Riemann surface $M$ of genus $g$ with a canonical homology basis $\Sigma$ and a triangulation $K, \Lambda_{K}$ lies in the Siegel upper half space.

\section{Main results}

In this section, we study the relations between holomorphic 1-forms and holomorphic 1-cochains. More precisely, we prove that holomorphic 1-cochains provide an approximation of holomorphic 1-forms. First of all, we introduce a correspondence between holomorphic 1-forms and holomorphic 1-cochains as follows.

Definition 5.1. For $\omega \in \mathscr{H} \Omega^{1,0}(M)$, we define $\imath_{\omega} \in \mathscr{H} C^{1,0}(K)$ which satisfies

$$
\iota_{\omega}\left(a_{j}\right)=\int_{a_{j}} \omega
$$

for $1 \leq j \leq g$.

Lemma 5.2. The map $\imath: \mathscr{H} \Omega^{1,0}(M) \rightarrow \mathscr{H} C^{1,0}(K)$ defined by $\omega \mapsto \imath_{\omega}$ is an isomorphism.

Proof. It is clear from the following diagram

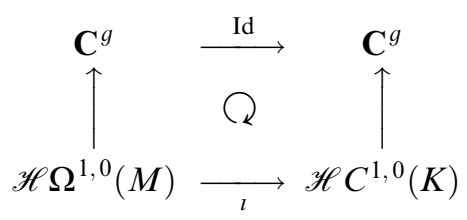

where the isomorphisms from $\mathscr{H} \Omega^{1,0}(M)$ to $\mathbf{C}^{g}$ and from $\mathscr{H} C^{1,0}(K)$ to $\mathbf{C}^{g}$ are as follows:

$$
\mathscr{H} \mathbf{\Omega}^{1,0}(M) \ni \omega \mapsto\left(\int_{a_{1}} \omega, \ldots, \int_{a_{g}} \omega\right) \in \mathbf{C}^{g}
$$

and

$$
\mathscr{H} C^{1,0}(K) \ni \sigma \mapsto\left(\sigma\left(a_{1}\right), \ldots, \sigma\left(a_{g}\right)\right) \in \mathbf{C}^{g}
$$

For this map $\omega \mapsto \imath_{\omega}$, we show the following theorem which is the main result of this paper.

THEOREM 5.3. Let $M$ be a closed Riemann surface of genus $g$ with a canonical homology basis $\Sigma, \omega$ an arbitrary holomorphic 1-form on $M$. In the case of $g=1$, for any triangulation $K$ of $M$, we have

$$
\left\|W l_{\omega}-\omega\right\|_{\Omega}=0 .
$$


In the case of $g>1$, for any sequence $\left\{K_{n}\right\}_{n \in \mathbf{N}}$ of triangulations of $M$ with the mesh converging to zero, we have

$$
\lim _{n \rightarrow \infty}\left\|W l_{\omega}^{n}-\omega\right\|_{\Omega}=0
$$

where $l_{\omega}^{n} \in \mathscr{H} C^{1,0}\left(K_{n}\right)$.

To prove this theorem, we need to study some relations between holomorphic 1-cochains and holomorphic 1-forms. In the following theorem, we give an estimation of $\mathscr{L}^{2}$-distance between holomorphic 1 -forms and holomorphic 1-cochains for a fixed triangulation.

THEOREM 5.4. Let $M$ be a closed Riemann surface of genus $g$ with a canonical homology basis $\Sigma$ and a triangulation $K$, and let $\left\{\theta_{1}, \ldots, \theta_{g}\right\}$ be the canonical basis of $\mathscr{H} \Omega^{1,0}(M),\left\{\sigma_{1}, \ldots, \sigma_{q}\right\}$ the canonical basis of $\mathscr{H} C^{1,0}(K)$ and $\Pi_{K}=\left(\pi_{j k}^{K}\right)_{1 \leq j, k \leq g}$ the combinatorial period matrix. Then there exists a vector $\Phi_{K}=\left(\varphi_{1}, \ldots, \varphi_{g}\right) \in(0,1]^{g}$ such that

In addition, we have

$$
\left\langle\star \sigma_{j}, \sigma_{j}\right\rangle_{C}=\left\langle-i \varphi_{j} \sigma_{j}, \sigma_{j}\right\rangle_{C} .
$$

and

$$
\left\|W \sigma_{j}-\theta_{j}\right\|_{\Omega}=\sqrt{2 \operatorname{Im} \pi_{j j}^{K}\left(\frac{1}{\varphi_{j}}-1\right)},
$$

$$
\left\|W l_{\omega}-\omega\right\|_{\Omega} \leq \sum_{j=1}^{g}\left|\int_{a_{j}} \omega\right| \cdot \sqrt{2 \operatorname{Im} \pi_{j j}^{K}\left(\frac{1}{\varphi_{j}}-1\right)},
$$

for all $\omega \in \mathscr{H} \Omega^{1,0}(M)$.

Proof. By Theorem 4.12, we have

$$
\operatorname{Im} \Pi=-\operatorname{Im} \Pi_{K}+\operatorname{Im} \Lambda_{K}
$$

and

$$
\operatorname{Im} \pi_{j j}=-\operatorname{Im} \pi_{j j}^{K}+\operatorname{Im}\left\langle W \sigma_{j}, \star \theta_{j}\right\rangle_{\Omega},
$$

for $1 \leq j \leq g$. Using Riemann's bi-linear relation, we compute

$$
\begin{aligned}
\left\langle\theta_{j}, \theta_{j}\right\rangle_{\Omega} & =i\left\langle-i \theta_{j}, \theta_{j}\right\rangle_{\Omega} \\
& =i\left\langle\star \theta_{j}, \theta_{j}\right\rangle_{\Omega} \\
& =i \sum_{k=1}^{g}\left(\int_{a_{k}} \theta_{j} \int_{b_{k}} \overline{\theta_{j}}-\int_{b_{k}} \theta_{j} \int_{a_{k}} \overline{\theta_{j}}\right) \\
& =i\left(\overline{\pi_{j j}}-\pi_{j j}\right) \\
& =2 \operatorname{Im} \pi_{j j} .
\end{aligned}
$$


Similary, we obtain $i\left\langle\star \sigma_{j}, \sigma_{j}\right\rangle_{C}=2 \operatorname{Im} \pi_{j j}^{K}$. Also we compute

$$
\begin{aligned}
2 \operatorname{Im}\left\langle W \sigma_{j}, \star \theta_{j}\right\rangle_{\Omega} & =i\left(\overline{\left\langle W \sigma_{j}, \star \theta_{j}\right\rangle_{\Omega}}-\left\langle W \sigma_{j}, \star \theta_{j}\right\rangle_{\Omega}\right) \\
& =i\left(\left\langle\star \theta_{j}, W \sigma_{j}\right\rangle_{\Omega}-\left\langle W \sigma_{j}, \star \theta_{j}\right\rangle_{\Omega}\right) \\
& =\left\langle\theta_{j}, W \sigma_{j}\right\rangle_{\Omega}+\left\langle W \sigma_{j}, \theta_{j}\right\rangle_{\Omega} .
\end{aligned}
$$

By (5.1),

$$
\left\langle\theta_{j}, \theta_{j}\right\rangle_{\Omega}-\left\langle\theta_{j}, W \sigma_{j}\right\rangle_{\Omega}-\left\langle W \sigma_{j}, \theta_{j}\right\rangle_{\Omega}=-i\left\langle\star \sigma_{j}, \sigma_{j}\right\rangle_{C} .
$$

Then we have

$$
\left\|W \sigma_{j}-\theta_{j}\right\|_{\Omega}^{2}=\left\langle W \sigma_{j}-\theta_{j}, W \sigma_{j}-\theta_{j}\right\rangle_{\Omega}=\left\langle\sigma_{j}, \sigma_{j}\right\rangle_{C}-i\left\langle\star \sigma_{j}, \sigma_{j}\right\rangle_{C} .
$$

Here we define $\varphi_{j}$ by

$$
\varphi_{j}=1-\left(\frac{\left\|W \sigma_{j}-\theta_{j}\right\|_{\Omega}}{\left\|\sigma_{j}\right\|_{C}}\right)^{2}
$$

By this definition,

$$
\left\langle\sigma_{j}, \sigma_{j}\right\rangle_{C}-i\left\langle\star \sigma_{j}, \sigma_{j}\right\rangle_{C}=\left(1-\varphi_{j}\right)\left\langle\sigma_{j}, \sigma_{j}\right\rangle_{C},
$$

and therefore

$$
\left\langle\star \sigma_{j}, \sigma_{j}\right\rangle_{C}=\left\langle-i \varphi_{j} \sigma_{j}, \sigma_{j}\right\rangle_{C} .
$$

Since $\Pi_{K}$ is an element of the Siegel upper half space, the diagonal elements $\operatorname{Im} \pi_{j j}^{K}(1 \leq j \leq g)$ of $\operatorname{Im} \Pi_{K}$ are all positive. Thus, by (5.2),

$$
\left\|\sigma_{j}\right\|_{C}^{2}-\left\|W \sigma_{j}-\theta_{j}\right\|_{\Omega}^{2}=i\left\langle\star \sigma_{j}, \sigma_{j}\right\rangle_{C}=2 \operatorname{Im} \pi_{j j}^{K}>0,
$$

and so

$$
0 \leq \frac{\left\|W \sigma_{j}-\theta_{j}\right\|_{\Omega}^{2}}{\left\|\sigma_{j}\right\|_{C}^{2}}<1
$$

This implies that $0<\varphi_{j} \leq 1 \quad(1 \leq j \leq g)$ and therefore $\Phi_{K} \in(0,1]^{g}$. By the definition of $\varphi_{j}$,

$$
\left\|W \sigma_{j}-\theta_{j}\right\|_{\Omega}=\sqrt{1-\varphi_{j}}\left\|\sigma_{j}\right\|_{C}
$$

Since

$$
\left\|\sigma_{j}\right\|_{C}^{2}=\frac{i\left\langle\star \sigma_{j}, \sigma_{j}\right\rangle_{C}}{\varphi_{j}}=\frac{2 \operatorname{Im} \pi_{j j}^{K}}{\varphi_{j}}
$$

and $\left\|\sigma_{j}\right\|_{C}>0$, we have

$$
\left\|W \sigma_{j}-\theta_{j}\right\|_{\Omega}=\sqrt{1-\varphi_{j}}\left\|\sigma_{j}\right\|_{C}=\sqrt{1-\varphi_{j}} \cdot \sqrt{\frac{2 \operatorname{Im} \pi_{j j}^{K}}{\varphi_{j}}}=\sqrt{2 \operatorname{Im} \pi_{j j}^{K}\left(\frac{1}{\varphi_{j}}-1\right)} .
$$


For $\omega \in \mathscr{H} \Omega^{1,0}(M)$ and $\imath_{\omega} \in \mathscr{H} C^{1,0}(K)$, we may write

$$
\omega=\sum_{j=1}^{g}\left(\int_{a_{j}} \omega\right) \cdot \theta_{j}
$$

and

$$
\iota_{\omega}=\sum_{j=1}^{g}\left(\int_{a_{j}} \omega\right) \cdot \sigma_{j} .
$$

Hence we conclude

$$
\begin{aligned}
\left\|W \boldsymbol{l}_{\omega}-\omega\right\|_{\Omega} & =\left\|\sum_{j=1}^{g}\left(\int_{a_{j}} \omega\right)\left(W \sigma_{j}-\theta_{j}\right)\right\|_{\Omega} \\
& \leq \sum_{j=1}^{g}\left|\int_{a_{j}} \omega\right| \cdot\left\|W \sigma_{j}-\theta_{j}\right\|_{\Omega} \\
& =\sum_{j=1}^{g}\left|\int_{a_{j}} \omega\right| \cdot \sqrt{2 \operatorname{Im} \pi_{j j}^{K}\left(\frac{1}{\varphi_{j}}-1\right) .}
\end{aligned}
$$

Next we study the behavior of $\Phi_{K}$. In the case of genus 1 , we show that $\Phi_{K}=\varphi_{1}=1$. This implies that for any triangulation $K$ of a complex torus, $\mathscr{H} C^{1,0}(K)$ is the eigenspace of $\star$ for the eigenvalue $-i$, i.e.,

$$
\mathscr{H} C^{1,0}(K)=\left\{\sigma \in \mathscr{H} C^{1}(K) \mid \star \sigma=-i \sigma\right\} .
$$

To prove this, we show the following lemma which is a characterization of $\Phi_{K}=(1, \ldots, 1)$.

Lemma 5.5. Let $M$ be a closed Riemann surface with a canonical homology basis $\Sigma$ and a triangulation $K$, and let $\left\{\theta_{1}, \ldots, \theta_{g}\right\}$ be the canonical basis of $\mathscr{H} \Omega^{1,0}(M)$. Let $\Phi_{K}$ be the vector as in Theorem 5.4. Then the following three conditions are equivalent:

(a) $\Phi_{K}=(1, \ldots, 1)$.

(b) $\mathscr{H} C^{1,0}(K)=\left\{\sigma \in \mathscr{H} C^{1}(K) \mid \star \sigma=-i \sigma\right\}$.

(c) $W R \theta_{j}=\theta_{j}$ a.e. on $M$ for all $j$.

Proof. $\quad(\mathrm{a}) \Rightarrow(\mathrm{c})$ : By Theorem 5.4, we obtain

$$
\left\|W \sigma_{j}-\theta_{j}\right\|_{\Omega}=0,
$$

and so $W \sigma_{j}=\theta_{j}$ a.e. on $M$. By Theorem 2.10; $R W=I d$, we have

$$
W R \theta_{j}=W R W \sigma_{j}=W \sigma_{j}=\theta_{j}
$$

a.e. on $M$. 
(c) $\Rightarrow\left(\right.$ b): For any $\sigma \in C^{1}(K)$, we compute

$$
\begin{aligned}
\left\langle\star R \theta_{j}, \sigma\right\rangle_{C} & =\iint_{M} W R \theta_{j} \wedge \overline{W \sigma} \\
& =\iint_{M} \theta_{j} \wedge \overline{W \sigma} \\
& =\left\langle\star \theta_{j}, W \sigma\right\rangle_{\Omega} \\
& =-i\left\langle\theta_{j}, W \sigma\right\rangle_{\Omega} \\
& =-i\left\langle W R \theta_{j}, W \sigma\right\rangle_{\Omega} \\
& =-i\left\langle R \theta_{j}, \sigma\right\rangle_{\Omega} \\
& =\left\langle-i R \theta_{j}, \sigma\right\rangle_{\Omega} .
\end{aligned}
$$

This implies that $\star R \theta_{j}=-i R \theta_{j}$. By Lemma 2.9, we have

$$
\delta R \theta_{j}=R d \theta_{j}=0,
$$

and by Lemma $2.15(1)$,

$$
\delta^{*} R \theta_{j}=i \delta^{*} \star R \theta_{j}=i \star \delta R \theta_{j}=0 .
$$

Thus all $R \theta_{j}(1 \leq j \leq g)$ are harmonic 1 -cochains which have eigenvalues $-i$ of $\star$, and therefore they are holomorphic 1-cochains. Since $\left\{\theta_{1}, \ldots, \theta_{g}\right\}$ is a basis of $\mathscr{H} \Omega^{1,0}(M)$ and $W R=I d,\left\{R \theta_{1}, \ldots, R \theta_{g}\right\}$ is a basis of $\mathscr{H} C^{1,0}(K)$. Since every eigenvalue of $R \theta_{j}$ is $-i$,

$$
\mathscr{H} C^{1,0}(K)=\left\{\sigma \in \mathscr{H} C^{1}(K) \mid \star \sigma=-i \sigma\right\} .
$$

Finally, it is clear that (b) induces (a).

THEOREM 5.6. Let $M$ be a closed Riemann surface of genus 1 (complex torus) with a canonical homology basis $\Sigma, K$ a triangulation of $M$, and $\varphi_{1} \in(0,1]$ which satisfies $\left\langle\star \sigma_{1}, \sigma_{1}\right\rangle_{C}=\left\langle-i \varphi_{1} \sigma_{1}, \sigma_{1}\right\rangle_{C}$, where $\left\{\sigma_{1}\right\}$ is the canonical basis of $\mathscr{H} C^{1,0}(K)$. Then $\varphi_{1}=1$.

Proof. Since the canonical basis of $\mathscr{H} \Omega^{1,0}(M)$ is $\{c d z\}$ where $z$ is a local coordinate and $c$ is some complex number, by Lemma 5.5, it is enough to show that $W R d z=d z$ a.e. on $M$.

First of all, we express $W R d z$ by

$$
W R d z=W\left(\sum_{\tau} R d z(\tau) \cdot \tau\right)=\sum_{\tau} R d z(\tau) \cdot W \tau,
$$

where the sum is taken over all 1 -simplices $\tau$ of $K$. Let $\left[p_{0}, p_{1}, p_{2}\right]$ be any 2 -simplex of $K$ with the barycentric coordinates $\mu_{0}, \mu_{1}$ and $\mu_{2}$. Since $W \tau=0$ on $M \backslash \overline{S t(\tau)}$, on the interior of $\left[p_{0}, p_{1}, p_{2}\right]$, we compute 


$$
\begin{aligned}
W R d z= & R d z\left(\left[p_{0}, p_{1}\right]\right) \cdot W\left[p_{0}, p_{1}\right]+R d z\left(\left[p_{1}, p_{2}\right]\right) \cdot W\left[p_{1}, p_{2}\right] \\
& +R d z\left(\left[p_{2}, p_{0}\right]\right) \cdot W\left[p_{2}, p_{0}\right] \\
= & \left(\int_{\left[p_{0}, p_{1}\right]} d z\right) \cdot W\left[p_{0}, p_{1}\right]+\left(\int_{\left[p_{1}, p_{2}\right]} d z\right) \cdot W\left[p_{1}, p_{2}\right] \\
& +\left(\int_{\left[p_{2}, p_{0}\right]} d z\right) \cdot W\left[p_{2}, p_{0}\right] \\
= & \left(p_{1}-p_{0}\right) \cdot W\left[p_{0}, p_{1}\right]+\left(p_{2}-p_{1}\right) \cdot W\left[p_{1}, p_{2}\right]+\left(p_{0}-p_{2}\right) \cdot W\left[p_{2}, p_{0}\right] \\
= & \left(p_{1}-p_{0}\right) \cdot\left(\mu_{0} d \mu_{1}-\mu_{1} d \mu_{0}\right)+\left(p_{2}-p_{1}\right) \cdot\left(\mu_{1} d \mu_{2}-\mu_{2} d \mu_{1}\right) \\
& +\left(p_{0}-p_{2}\right) \cdot\left(\mu_{2} d \mu_{0}-\mu_{0} d \mu_{2}\right) \\
= & p_{0}\left(\mu_{2} d \mu_{0}-\mu_{0} d \mu_{2}-\mu_{0} d \mu_{1}+\mu_{1} d \mu_{0}\right) \\
& +p_{1}\left(\mu_{0} d \mu_{1}-\mu_{1} d \mu_{0}-\mu_{1} d \mu_{2}+\mu_{2} d \mu_{1}\right) \\
& +p_{2}\left(\mu_{1} d \mu_{2}-\mu_{2} d \mu_{1}-\mu_{2} d \mu_{0}+\mu_{0} d \mu_{2}\right)
\end{aligned}
$$

On the interior of $\left[p_{0}, p_{1}, p_{2}\right](\ni z)$, the barycentric coordinates satisfy

$$
\mu_{0}(z)+\mu_{1}(z)+\mu_{2}(z)=1
$$

and

$$
d \mu_{0}+d \mu_{1}+d \mu_{2}=0 .
$$

Since $z=\sum_{j=0}^{2} p_{j} \mu_{j}(z)$, we have

$$
W R d z=\sum_{j=0}^{2} p_{j} \cdot d \mu_{j}(z)=d\left(\sum_{j=0}^{2} p_{j} \mu_{j}(z)\right)=d z .
$$

On the other hand, the union of the sets of all vertices ( 0 -simplices) and 1 -simplices of $K$ is a finite set and therefore it is a null set. Hence $W R d z=d z$ a.e. on $M$. By Lemma 5.5, we conclude that $\varphi_{1}=1$.

Finally we consider the behavior of $\Phi_{K}$ for higher genus. The following theorem implies that eigenvalues of $\star$ on $\mathscr{H} C^{1,0}(K)$ converge to $-i$, as the mesh tends to zero.

THEOREM 5.7. Let $M$ be a closed Riemann surface of genus $g>1$ with a canonical homology basis $\Sigma$ and $\left\{K_{n}\right\}_{n \in \mathbf{N}}$ be a sequence of triangulations of $M$ with the mesh converging to zero. Let $\Phi_{K_{n}}=\left(\varphi_{1}^{n}, \ldots, \varphi_{g}^{n}\right)$ be the vector in $(0,1]^{g}$ such that

$$
\left\langle\star \sigma_{j}^{n}, \sigma_{j}^{n}\right\rangle_{C}=\left\langle-i \varphi_{j}^{n} \sigma_{j}^{n}, \sigma_{j}^{n}\right\rangle_{C},
$$


where each $\left\{\sigma_{1}^{n}, \ldots, \sigma_{g}^{n}\right\}$ be the canonical basis of $\mathscr{H} C^{1,0}\left(K_{n}\right)$. Then we have

$$
\lim _{n \rightarrow \infty} \Phi_{K_{n}}=(1, \ldots, 1) \text {. }
$$

Proof. Let $\left\{\omega_{1}, \ldots, \omega_{g}\right\}$ be an orthogonal basis of $\mathscr{H} \Omega^{1,0}(M)$ and $R^{n}$ the de Rham map from $\Omega(M)$ to $C\left(K_{n}\right)$. By the Hodge decomposition and $\mathscr{H} C^{1}\left(K_{n}\right)=$ $\mathscr{H} C^{1,0}\left(K_{n}\right) \oplus \mathscr{H} C^{0,1}\left(K_{n}\right)$, we obtain

$$
R^{n} \omega_{j}=\delta^{*} k_{j}^{n}+h_{j}^{n}+\tilde{h}_{j}^{n}+\delta g_{j}^{n}
$$

for any $n \in \mathbf{N}$, where $h_{j}^{n} \in \mathscr{H} C^{1,0}\left(K_{n}\right)$ and $\tilde{h}_{j}^{n} \in \mathscr{H} C^{0,1}\left(K_{n}\right)$.

First, we show that the number of $K_{n}$, such that $\left\{h_{1}^{n}, \ldots, h_{q}^{n}\right\}$ is not a basis of $\mathscr{H} C^{1,0}\left(K_{n}\right)$, is finite. Now we assume that the number is infinite. Then there exist $j \in\{1, \ldots, g\}$ and a subsequence $\left\{K_{m}\right\}$ of $\left\{K_{n}\right\}$ such that each $h_{j}^{m}$ is generated by the other elements, i.e.,

$$
h_{j}^{m}=\sum_{p \neq j} c_{j p}^{m} h_{p}^{m},
$$

for all $m \in \mathbf{N}$, where $c_{j p}^{m} \in \mathbf{C}$. Since $h_{j}^{m}-\sum_{p \neq j} c_{j p}^{m} h_{p}^{m}$ is the holomorphic part of $R^{m}\left(\omega_{j}-\sum_{p \neq j} c_{j p}^{m} \omega_{p}\right)$, by Lemma 4.9 , we have

$$
\lim _{m \rightarrow \infty}\left\|\omega_{j}-\sum_{p \neq j} c_{j p}^{m} \omega_{p}\right\|_{\Omega}=0 .
$$

Also, since $\left\{\omega_{1}, \ldots, \omega_{g}\right\}$ is an orthogonal basis,

$$
\left\|\omega_{j}-\sum_{p \neq j} c_{j p}^{m} \omega_{p}\right\|_{\Omega}^{2}=\left\|\omega_{j}\right\|_{\Omega}^{2}+\sum_{p \neq j}\left|c_{j p}^{m}\right|^{2}\left\|\omega_{p}\right\|_{\Omega}^{2},
$$

and therefore

$$
0 \leq\left|c_{j p}^{m}\right|^{2}\left\|\omega_{p}\right\|_{\Omega}^{2} \leq\left\|\omega_{j}-\sum_{p \neq j} c_{j p}^{m} \omega_{p}\right\|_{\Omega}^{2} .
$$

By (5.3), we obtain $\lim _{m \rightarrow \infty}\left|c_{j p}^{m}\right|=0$ and (5.4) implies that $\left\|\omega_{j}\right\|_{\Omega}=0$. This is a contradiction since $\left\{\omega_{1}, \ldots, \omega_{g}\right\}$ is a basis.

Here we assume that $\left\{h_{1}^{n}, \ldots, h_{g}^{n}\right\}$ is a basis of $\mathscr{H} C^{1,0}\left(K_{n}\right)$ for all $n \in \mathbf{N}$. Then, for any $n \in \mathbf{N}$, we may write

$$
\sigma_{j}^{n}=\sum_{\ell=1}^{g} \tilde{c}_{j \ell}^{n} h_{\ell}^{n},
$$

for $1 \leq j \leq g$, where $\tilde{c}_{j \ell}^{n} \in \mathbf{C}$. Next we consider $\lim _{n \rightarrow \infty} \tilde{c}_{j \ell}^{n}$. Let $\left(\tilde{d}_{\ell j}^{n}\right)_{1 \leq \ell, j \leq g}$ be the inverse matrix of $\left(\tilde{c}_{j \ell}^{n}\right)_{1 \leq j, \ell \leq g}$. This matrix provides

$$
h_{\ell}^{n}=\sum_{j=1}^{g} \tilde{d}_{\ell j}^{n} \sigma_{j}^{n}
$$


and

$$
h_{\ell}^{n}\left(a_{k}\right)=\sum_{j=1}^{g} \tilde{d}_{\ell j}^{n} \sigma_{j}^{n}\left(a_{k}\right)=\sum_{j=1}^{g} \tilde{d}_{\ell j}^{n} \cdot \delta_{j k}=\tilde{d}_{\ell k}^{n},
$$

for $1 \leq \ell, k \leq g$. By Lemma 4.9 and 4.11, we have

$$
\lim _{n \rightarrow \infty} \tilde{d}_{\ell k}^{n}=\lim _{n \rightarrow \infty} h_{\ell}^{n}\left(a_{k}\right)=\lim _{n \rightarrow \infty} R W h_{\ell}^{n}\left(a_{k}\right)=\int_{a_{k}} \omega_{\ell},
$$

for $1 \leq \ell, k \leq g$. Note that each $W h_{\ell}^{n}$ is neither smooth nor closed, but it can be approximated by a sequence of closed smooth forms and therefore we may apply Lemma 4.11 to $W h_{\ell}^{n}$. Thus (5.5) implies that the matrix $\left(\tilde{d}_{\ell j}^{n}\right)_{1 \leq \ell, j \leq g}$ converges to $\left(\int_{a_{j}} \omega_{\ell}\right)_{1 \leq \ell, j \leq g}$, and therefore $\left(\tilde{c}_{j \ell}^{n}\right)_{1 \leq j, \ell \leq g}=\left(\tilde{d}_{\ell j}^{n}\right)_{1 \leq \ell, j \leq g}^{-1}$ also converges to a matrix $\left(s_{j \ell}\right)_{1 \leq j, p \leq g}$, where each $s_{j \ell}$ is determined by $\int_{a_{1}} \omega_{1}, \ldots, \int_{a_{g}} \omega_{1}, \ldots$, $\int_{a_{1}} \omega_{g}, \ldots, \int_{a_{g}} \omega_{g}$.

Using the Cauchy-Schwarz inequality, we compute

$$
\begin{aligned}
0 \leq\left(1-\varphi_{j}^{n}\right)\left\|\sigma_{j}^{n}\right\|_{C}^{2} & =\left\langle\sigma_{j}^{n}, \sigma_{j}^{n}\right\rangle_{C}-i\left\langle-i \varphi_{j}^{n} \sigma_{j}^{n}, \sigma_{j}^{n}\right\rangle_{C} \\
& =\left\langle\sigma_{j}^{n}, \sigma_{j}^{n}\right\rangle_{C}-i\left\langle\star \sigma_{j}^{n}, \sigma_{j}^{n}\right\rangle_{C} \\
& =\left\langle\sigma_{j}^{n}-i \star \sigma_{j}^{n}, \sigma_{j}^{n}\right\rangle_{C} \\
& \leq\left\|\sigma_{j}^{n}-i \star \sigma_{j}^{n}\right\|_{C} \cdot\left\|\sigma_{j}^{n}\right\|_{C},
\end{aligned}
$$

and then

$$
0 \leq\left(1-\varphi_{j}^{n}\right)\left\|\sigma_{j}^{n}\right\|_{C} \leq\left\|\sigma_{j}^{n}-i \star \sigma_{j}^{n}\right\|_{C} .
$$

Since $\sigma_{j}^{n}=\sum_{\ell=1}^{g} \tilde{c}_{\ell j}^{n} h_{\ell}^{n}$, we have

$$
\begin{aligned}
\left\|\sigma_{j}^{n}-i \star \sigma_{j}^{n}\right\|_{C} & =\left\|\sum_{\ell=1}^{g} \tilde{c}_{j \ell}^{n}\left(h_{\ell}^{n}-i \star h_{\ell}^{n}\right)\right\|_{C} \\
& \leq \sum_{\ell=1}^{g}\left|\tilde{c}_{j \ell}^{n}\right| \cdot\left\|W h_{\ell}^{n}-i W \star h_{\ell}^{n}\right\|_{\Omega} \\
& =\sum_{\ell=1}^{g}\left|\tilde{c}_{j \ell}^{n}\right| \cdot\left\|W h_{\ell}^{n}-\omega_{\ell}+i \star \omega_{\ell}-i W \star h_{\ell}^{n}\right\|_{\Omega} \\
& \leq \sum_{\ell=1}^{g}\left|\tilde{c}_{j \ell}^{n}\right| \cdot\left(\left\|W h_{\ell}^{n}-\omega_{\ell}\right\|_{\Omega}+\left\|\star \omega_{\ell}-W \star h_{\ell}^{n}\right\|_{\Omega}\right) .
\end{aligned}
$$

Note that any holomorphic 1-form $\omega$ satisfies $\omega-i \star \omega=0$.

By Lemma 4.9 and 4.10 , there exist positive constants $C_{\ell}$, independent of $\left\{K_{n}\right\}$, such that

$$
\left\|W h_{\ell}^{n}-\omega_{\ell}\right\|_{\Omega}+\left\|W \star h_{\ell}^{n}-\star \omega_{\ell}\right\|_{\Omega} \leq C_{\ell} \cdot \eta\left(K_{n}\right) .
$$


Thus we have

$$
\left\|\sigma_{j}^{n}-i \star \sigma_{j}^{n}\right\|_{C} \leq \sum_{\ell=1}^{g}\left|\tilde{c}_{j \ell}^{n}\right| \cdot C_{\ell} \cdot \eta\left(K_{n}\right),
$$

and

$$
\lim _{n \rightarrow \infty}\left\|\sigma_{j}^{n}-i \star \sigma_{j}^{n}\right\|_{C}=0 .
$$

Namely, by (5.6), we have

$$
\lim _{n \rightarrow \infty}\left(1-\varphi_{j}^{n}\right)\left\|\sigma_{j}^{n}\right\|_{C}=0 .
$$

By Riemann's bi-linear relations and $0<\varphi_{j}^{n} \leq 1$,

$$
\left\|\sigma_{j}^{n}\right\|_{C}^{2}=\frac{i}{\varphi_{j}^{n}}\left\langle\star \sigma_{j}^{n}, \sigma_{j}^{n}\right\rangle_{C}=\frac{2 \operatorname{Im} \pi_{j j}^{K_{n}}}{\varphi_{j}^{n}} \geq 2 \operatorname{Im} \pi_{j j}^{K_{n}},
$$

and therefore $\lim _{n \rightarrow \infty}\left\|\sigma_{j}^{n}\right\|_{C}^{2} \geq 2 \operatorname{Im} \pi_{j j}>0$ by Theorem 4.8. Hence we conclude $\lim _{n \rightarrow \infty} \varphi_{j}^{n}=1$ for all $j$.

Combine Theorem 5.4, 5.6 and 5.7, we can easily show Theorem 5.3.

Acknowledgements. The author would like to thank Profs. Hiroshige Shiga and Masaharu Tanabe for many comments, suggestions and support on his work. He also would like to thank the referees for giving valuable suggestions.

\section{REFERENCES}

[1] J. Dodziuk, Finite-differrence apporoach to the Hodge theory of harmonic forms, Amer. J. of Math. 98 (1976), 79-104.

[2] J. Dodziuk and V. K. PAtodi, Riemannian structures and triangulations of manifolds, J. Indian Math. Soc. (N.S.) 40 (1976), 1-52.

[3] J. Dupont, Curvature and characteristic classesh, Lecture notes in mathematics 640, Springer-Verlag, 1978.

[ 4 ] B. EckmanN, Harmonische funktionnen und randvertanfgaben in einem komplex, Comment. Math. Helv. 17 (1944-45), 240-245.

[5] H. M. Farkas and I. Kra, Riemann surfaces, 2nd ed. Graduate texts in mathematics 71, Springer, New York, 1992.

[6] M. SpIVAK, A comprehensive introduction to differential geometry IV, Publish or Perish, Boston, MA, 1975.

[7] M. TAnabe, Several remarks on the combinatorial Hodge star, Topology Proc. 46 (2015), 33-43.

[8] H. Whitney, Geometric integration theory, Princeton Univ. Press, Princeton, NJ, 1957.

[9] S. O. Wilson, Cochain algebra on manifolds and convergence under refinement, Topology and Its Applications 154 (2007), 1898-1920.

[10] S. O. Wilson, Conformal cochains, Trans. Amer. Math. Soc. 360 (2008), 5247-5264. 
[11] S. O. Wilson, Addendum to "Conformal cochains," Trans. Amer. Math. Soc. 365 (2013), 5033-5033.

[12] D. YAmaki, A matrix equation on triangulated Riemann surfaces, Proc. Japan Acad, Ser. A. Math. Sci. 90 (2014), 37-42.

Daisuke Yamaki

Department of Mathematics

Tokyo Institute of TeChNology

OH-OKayama, Meguro-Ku

TOKYO 152-8551

JAPAN

E-mail: yamaki.d.aa@m.titech.ac.jp 